\title{
Genome-wide identification and functional characterization of the PheE2F/DP gene family in Moso bamboo
}

\author{
Long $\mathrm{Li}^{1}$, Qianqian Shi ${ }^{2}$, Zhouqi $\mathrm{Li}^{1}$ and Jian $\mathrm{Gao}^{3^{*}}$ (D)
}

\begin{abstract}
Background: E2F/DP proteins have been shown to regulate genes implicated in cell cycle control and DNA repair. However, to date, research into the potential role of the Moso bamboo E2F/DP family has been limited.

Results: Here, we identified 23 E2F/DPs in the Moso bamboo genome, including nine E2F genes, six DP genes, eight DEL genes and one gene with a partial E2F domain. An estimation of the divergence time of the paralogous gene pairs suggested that the E2F/DP family expansion primarily occurred through a whole-genome duplication event. A regulatory element and coexpression network analysis indicated that E2F/DP regulated the expression of cell cycle-related genes. A yeast two-hybrid assay and expression analysis based on transcriptome data and in situ hybridization indicated that the PheE2F-PheDP complex played important roles in winter Moso bamboo shoot growth. The qRT-PCR results showed that the PheE2F/DPs exhibited diverse expression patterns in response to drought and salt treatment and diurnal cycles.
\end{abstract}

Conclusion: Our findings provide novel insights into the Moso bamboo E2F/DP family and partial experimental evidence for further functional verification of the PheE2F/DPs.

Keywords: E2F/DPs, Moso bamboo, Expression, Cell cycle, Bamboo shoot

\section{Background}

Moso bamboo (Phyllostachys edulis) generates nearly 5 billion US dollars annually in economic value. It is the most important non-timber product used for commercial purposes in East Asia due to its fast growth rate [1, 2]. During Moso bamboo shoot growth, the height increase was created by simultaneous cell division and cell elongation. Cell division played critical roles during the winter and early growth periods, while cell elongation was predominant during the late growth period.

The E2F/DP transcription factors in higher plants are categorized into E2F, DP, and DEL (DP-E2F-like) groups based on their conserved domains [3]. The

\footnotetext{
*Correspondence: gaojianicbr@163.com; gaojian@icbr.ac.cn

${ }^{3}$ International Center for Bamboo and Rattan, Key Laboratory of Bamboo and Rattan Science and Technology, State Forestry Administration, Beijing, China Full list of author information is available at the end of the article
}

E2F group gene contains four functional domains including an RBR-binding domain, a 'marked box' domain, a DNA-binding domain, and a leucine zipper dimerization domain, while the members of the DP group lack the RBR-binding domain, a 'marked box' domain compared with E2F. The DEL group genes are considered to be atypical E2F/DPs and act in monomeric form, and the DEL genes only contain a duplicated DNA-binding domain [4].

The E2F/DP family has eight members in Arabidopsis. During cell proliferation, the canonical AtE2Fs reportedly play an antagonistic role, because E2Fc is a negative regulator, whereas E2Fa and E2Fb are positive regulators [5-7]. E2Fa and E2Fb interact with DPa to activate the cell cycle and cell proliferation-associated gene expression through the organization of their leucine zipper dimerization domains [8]. 
The E2F member might perform distinct roles in controlling the cell fate determination [3]. In mammals, the E2F signaling pathway is essential for cell growth and cell proliferation [9]. Studies on E2F in Arabidopsis have indicated that plants also have all the core regulators in the E2F signaling pathway, such as cyclin-dependent kinase inhibitors (CKIs), retinoblastoma (RBs), cyclins, and cyclin-dependent kinases (CDKs). In plants, E2F/DP binds to the E2FAT (TTTCCCGCC) motif in its target genes, playing a role in promoting transcription [10].

Previous studies identified 12 PhE2F/DP transcription factors from the first version of the Moso bamboo genome database [11]. However, because of limits in the draft genome sequence, a considerable number of PhE2F/DPs were still missing annotations. In addition, the expression changes and potential roles of the PheE2F/DP genes during bamboo shoot growth and in response to stress stimuli and diurnal cycles were still absent. In this study, a total of 23 E2F/DPs were identified based on the new version of the Moso bamboo genome annotation project, which provided chromosomelevel de novo genome assembly. The phylogenetic relationships, gene structures, and conserved motifs of 23 PheE2F/DP genes were analyzed. The promoter analysis indicated that various cis-acting elements, including light response and hormone signaling as well as many transcription factor binding sites, were present in the promoter region of the PheE2F genes. We surveyed potential gene promoters containing E2F/DP binding sites within the Moso bamboo genome and identified 580 E2F/DP target genes. Based on bioinformatic predictions, we further studied the expression dynamics of the PheE2F genes during bamboo shoot growth and in response to stress stimuli and diurnal cycles. A yeast twohybrid assay and an expression analysis were conducted to investigate the potential roles of PheE2F/DPs involved in Moso bamboo shoot growth. Our study provided new insights and some valuable information for the further functional verification of E2F/DPs in Moso bamboo.

\section{Results}

\section{Identification and classification of the PheE2F/DP gene} family

A total of 24 potential PheE2F/DP genes were identified in the Moso bamboo genome, including a PH02Gene25981.t1 with an incomplete E2F domain. To find whether the incomplete E2F domain in PH02Gene25981.t1 was caused by incorrect gene structure annotation in the bamboo genome or PH02Gene25981.t1 was merely just a pseudogene, we used three different methods to provide bioinformatic and experimental evidence. First, we used the CD-Search program from the NCBI to identified conserved domains involved in the whole PH02Gene25981.t1 sequence, including exons, introns, 5'-UTRs and 3'-UTRs, but no complete E2F_ $\mathrm{DD}$ or DP domains were identified in the PHO2Gene25981.t1 sequence. Second, we searched the expression information and potential splicing variants of PH02Gene25981.t1 in many previously published and unpublished transcriptome sequencing data, including single molecule sequencing data [2, 12]; however, no valuable information was identified. Lastly, we designed different primer pairs for PH02Gene25981.t1 CDS and the exon that contained the partial E2F domain. Different RNA samples were isolated from different tissues, including the leaves, culms, rhizome, bamboo shoots, seeds, roots, flowers, and seedlings under various abiotic stresses, which were used as clone templates, but we did not obtain the CDS sequence of PH02Gene25981.t1 from any of the tissues. Thus, we speculated that PH02Gene25981.t1 is just a pseudogene.

To determine the evolutionary relationship of E2F/ DPs in Moso bamboo and other model plants, a maximum likelihood tree of E2F/DP proteins from Moso bamboo, Oryza sativa, Brachypodium and Arabidopsis was constructed. For the 23 identified PheE2F/DP genes from Moso bamboo, the proteins could be classified into three functional groups: E2F, DP, and DEL [3]. Nine of these proteins were classified into the E2F group, six into the DP group and eight into the DEL group (Fig. 1a). In addition, a phylogenetic analysis of the E2F/DPs in four species indicated that PheE2F/DPs shared more sequence similarity with OsE2F/DPs and BdE2F/DPs than with AtE2F/DPs. For example, AT5G14960.1, AT3G01330.1, and AT3G486160.1 clustered together in the DEL group, showing a distant relationship to Brachypodium, rice, and Moso bamboo.

\section{Characterization of the PheE2F/DP gene family}

A gene structural analysis of the E2F/DP family revealed that the genes within the same group shared similar intron lengths and numbers (Fig. 1b). The number of introns in the DP and DEL groups ranged from seven to nine and three to nine, respectively. The average numbers of introns in the E2F group were much higher than those in the other two groups, and the intron quantity ranged from five to 16 .

Eight conserved motifs were identified in the E2F/ DPs using the MEME online program (Fig. 1c and Additional file 1: Fig. S1). Motifs 1 and 6 represent the E2F_TD domain, and motifs 2 and 3 represent the E2F_DD and DP domains, respectively. Motifs 2, 4 and 7 were found exclusively in the E2F group, whereas motifs 3 and 8 appeared only in the DP group. Most DEL members shared motifs 1 and 5 with most E2F protein sequences. The motif analysis indicated that proteins involved in the same group shared several identical motifs outside the conserved 

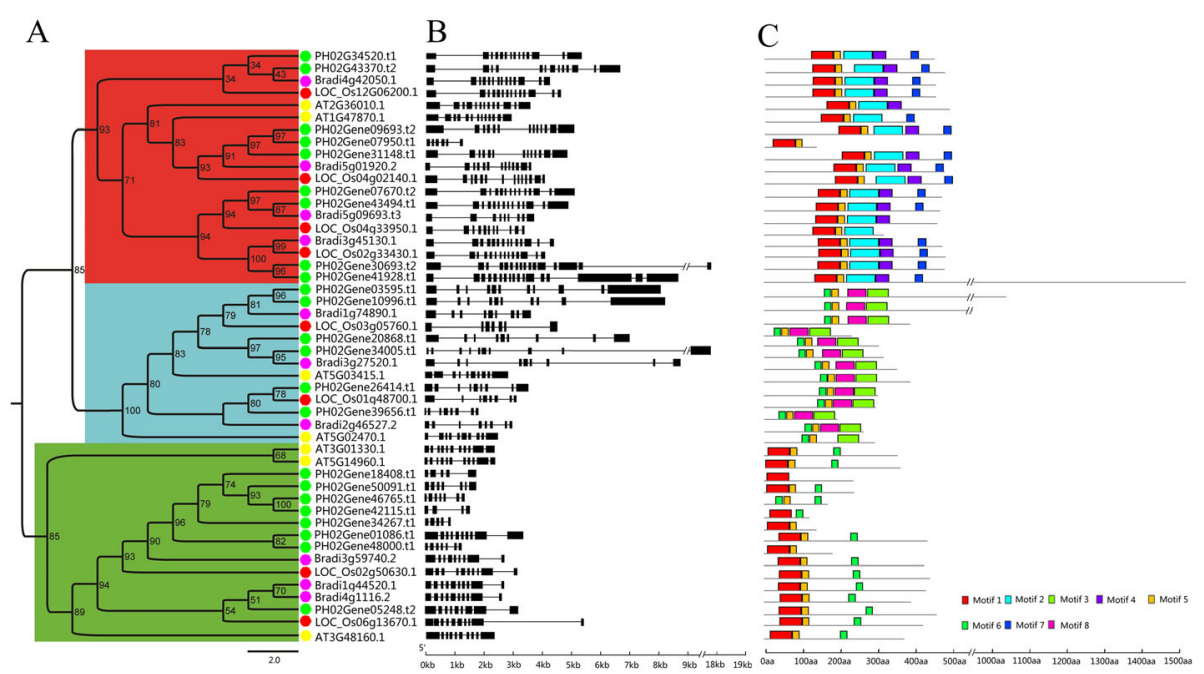

Fig. 1 Phylogenetic tree, gene structure and conserved motif compositions of the PheE2F/DP families. a Phylogenetic tree relationship of the E2F/DP family. The A. thaliana, O. sativa, B. distachyon, and Moso bamboo proteins are marked with yellow, red, purple, and green dots, respectively. $\mathbf{b}$ Gene structures of the E2F/DP genes. Black boxes and black lines represent exons and introns, respectively. Scale bar: $1.0 \mathrm{~kb}$. The -//- represents $8.0 \mathrm{~kb}$. c Distribution of conserved motifs within each group. The scale bar represents 100 amino acids

domain mentioned above, indicating functional conservations within the same group.

Gene duplication events play a critical role in the production of new functions and in gene expansion. Thus, we analyzed the potential duplication events of the PheE2F/DPs. No tandem duplication events were identified in the Moso bamboo E2F/DPs, but there were 16 genes pairing $12 \mathrm{E} 2 \mathrm{~F} / \mathrm{DP}$ paralogous pairs in synteny blocks in the Moso bamboo genome (Fig. 2a). For example, a large amount of highly conserved synteny blocks was observed between chromosomes (chr) 16 and 14, which contained Phe02Gene26414.t1 and Phe02Gene39656.t1, respectively (Fig. 2b). Similar phenomena were observed between chr 15 and 21, which contained Phe02Gene26414.t1 and Phe02Gene39656.t1, respectively, as well as other chromosome pairs that contained or did not contain paralogous E2F/DP pairs. Furthermore, we calculated the divergence time of the paralogous pairs (Additional file 1: Table S1). The divergence for most PheE2F/DP gene pairs (7 of 8) was approximately 6.5 to 13.5 mya, similar to the Moso bamboo whole-genome duplication event (7-12 mya) [13], and much later than those of $O$. sativa, $B$. distachyon and $A$. thaliana. These results suggested that a whole-genome duplication event played a critical role in the Moso bamboo gene expansion, including E2F/DPs.

\section{Regulatory network of PheE2F/DP in Moso bamboo}

The cis-elements, which are located in the promoter region, are essential to the spatial, temporal, and tissuespecific control of gene expression under external and internal environmental stimuli [14]. Thus, we screened the cis-element at the promoter regions (2000-bp upstream) of all the PheE2F/DP genes using the PlantCARE database. A large number of cis-elements including light response sites, hormone response sites, transcription factor binding sites and meristem-related motifs were found (Fig. 3). All 23 PheE2F/DP promoters contained at least one light response motif. MYB binding motifs were found in all 23 promoters of PheE2F/DP genes, and most PheE2F/DP promoters harbored MYC binding elements. Thirteen PheE2F/DP promoters contained P-box or GARE motifs that were responsive to gibberellin stimulation. The abscisic acid responsiveness (ABRE) site was abundant in the PheE2F/DP promoters, and this element was found in 21 promoters. In addition, the TGACG motif and TCA element, which are responsive to methyl jasmonate (MeJA) and salicylic acid stimulation, appeared in most of the PheE2F/DP promoter regions. Nineteen promoters contained CAT box, a meristem expression-related cis-element, indicating the importance of the PheE2Fs involved in cell division and proliferation. In addition, no significant difference was observed between different groups following a comparison of cis-acting elements.

A previous study reported that E2F/DP binds to the E2FAT (TTTCCCGCC) motif of its target genes and regulates their transcription [10]. Thus, we screened the binding sites in the promoter regions of 51,074 proteincoding genes in the Moso bamboo genome using PlantCARE. Finally, 580 genes that contained E2F/DP binding sites in their promoter regions were identified. To explore the potential function of the genes regulated by PheE2F/DP, we performed a Gene Ontology (GO) 


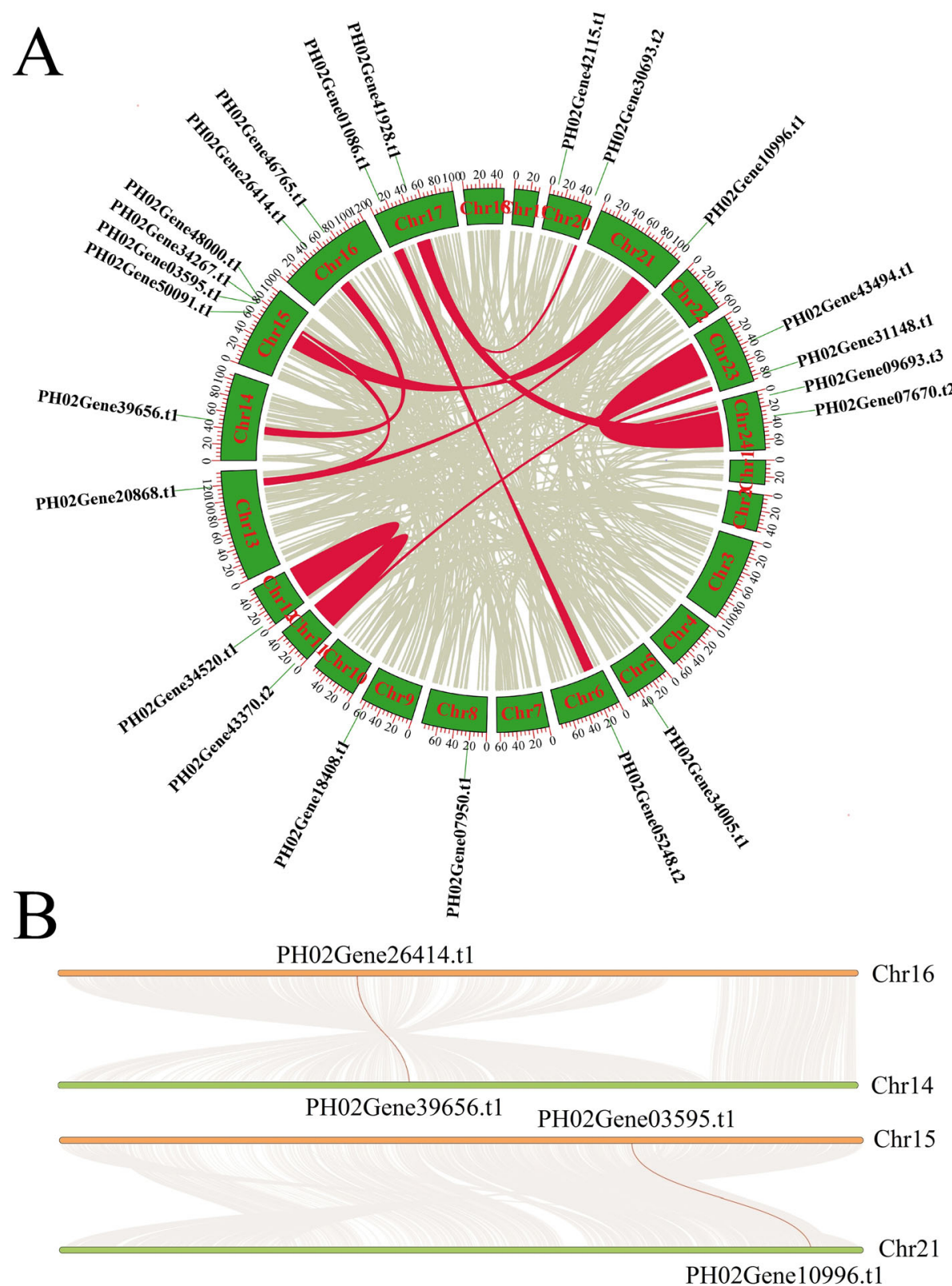

Fig. 2 Gene location and collinearity analysis of the PheE2F gene family. a Syntenic relationships among synteny blocks carrying the 11 duplicated PheE2F/DP gene pairs. Red bars denote synteny blocks that harbored duplicated E2F/DP genes. Grey bars in the background indicate all the identified synteny blocks. b Example of collinearity analysis between two Moso bamboo chromosomes. Grey lines in the background indicate all the duplicated genes between two chromosomes, and the pairs of duplicated PheE2F/DP genes are connected by red lines

enrichment analysis (Fig. 4). In the biological processes category, the groups with the highest abundance of genes included the mitotic cell cycle, mitotic cell cycle process, regulation of DNA metabolic process, regulation of DNA replication, and regulation of the cell cycle.

The transcriptome data generated from 13 different culm tissue samples were used to investigate the expression patterns of the PheE2F/DPs. Most E2F/DPs showed relatively high expression levels in winter bamboo shoots (S1) and spring bamboo shoots, especially during the early growth period (S2-S5) compared with the other growing culms, such as the rhizome (R) and seedling stems (SS1 and SS2). Of the 18 genes that were expressed in at least one tissue, 11 showed the highest expression level in winter bamboo shoots (S1) (Fig. 5). PH02Gene34520.t1 and PH02Gene43370.t2 were highly expressed in 50-cm-tall bamboo shoots (S2), while PHO2Gene46765.t1 exhibited the highest accumulation level in the outward rhizome (O). PHO2Gene05248.t2 and 

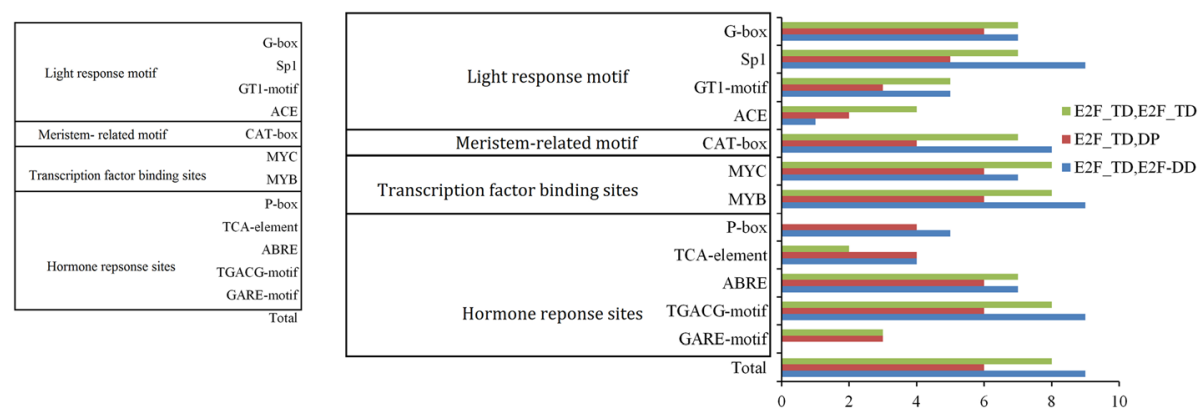

Fig. 3 The quantity of cis-regulatory elements (CRES) in the E2F/DP promoters. Only identified elements with greater than five CREs were retained

PH02Gene10996.t1 were highly expressed in S4 (300cm-tall bamboo shoots) and S5 (600-cm-tall bamboo shoots), respectively. The expression analysis results revealed that the PheE2F/DPs played important roles in the growth of the bamboo shoots, especially during the winter period.
To understand the regulatory network of PheE2F/DPs implicated in the different types of culm growth, we performed regulation tests between quantitative changes in the transcripts from the 13 different culm tissue samples. Based on the regulatory relationship obtained by predicting the regulatory elements in the promoter

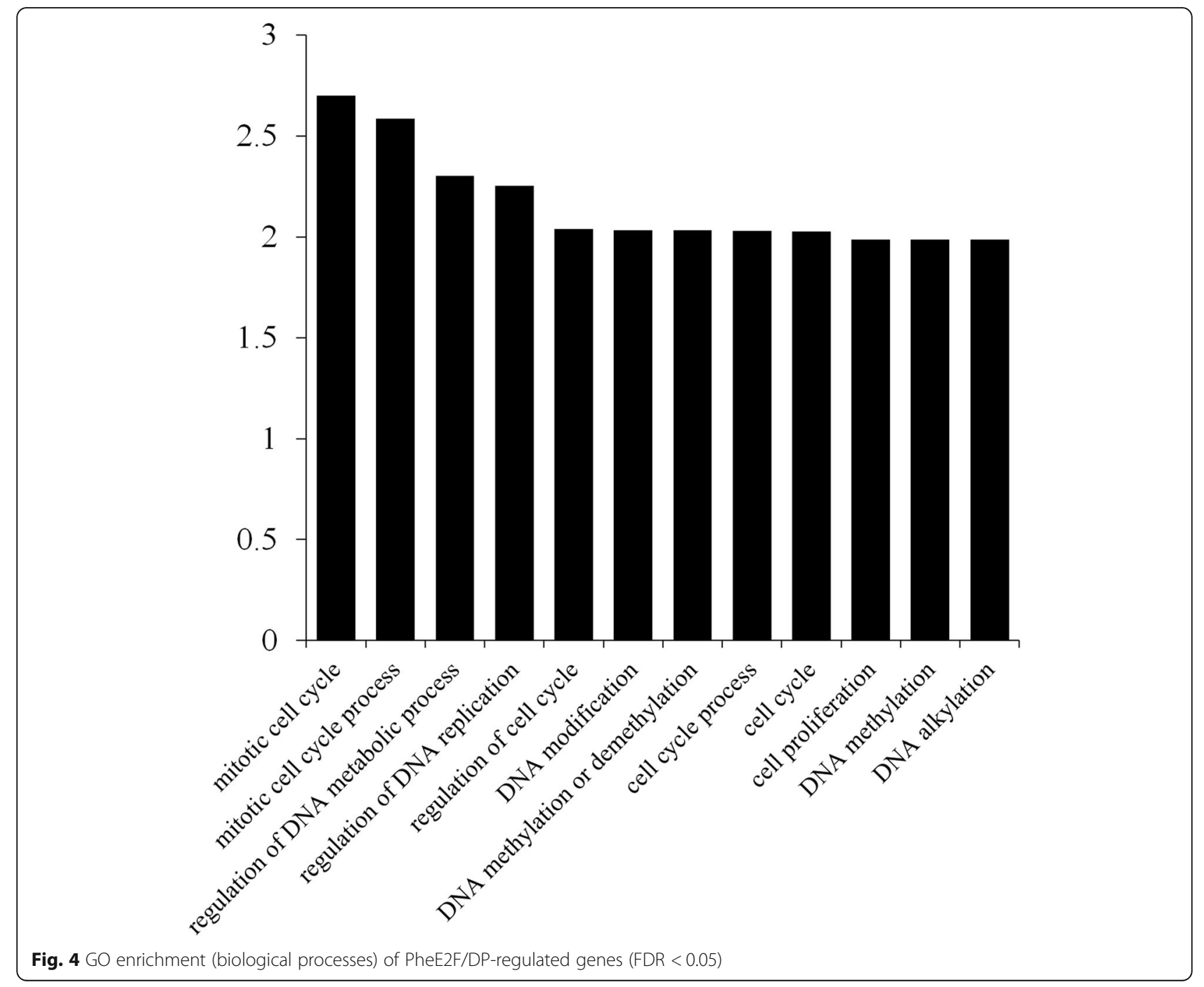




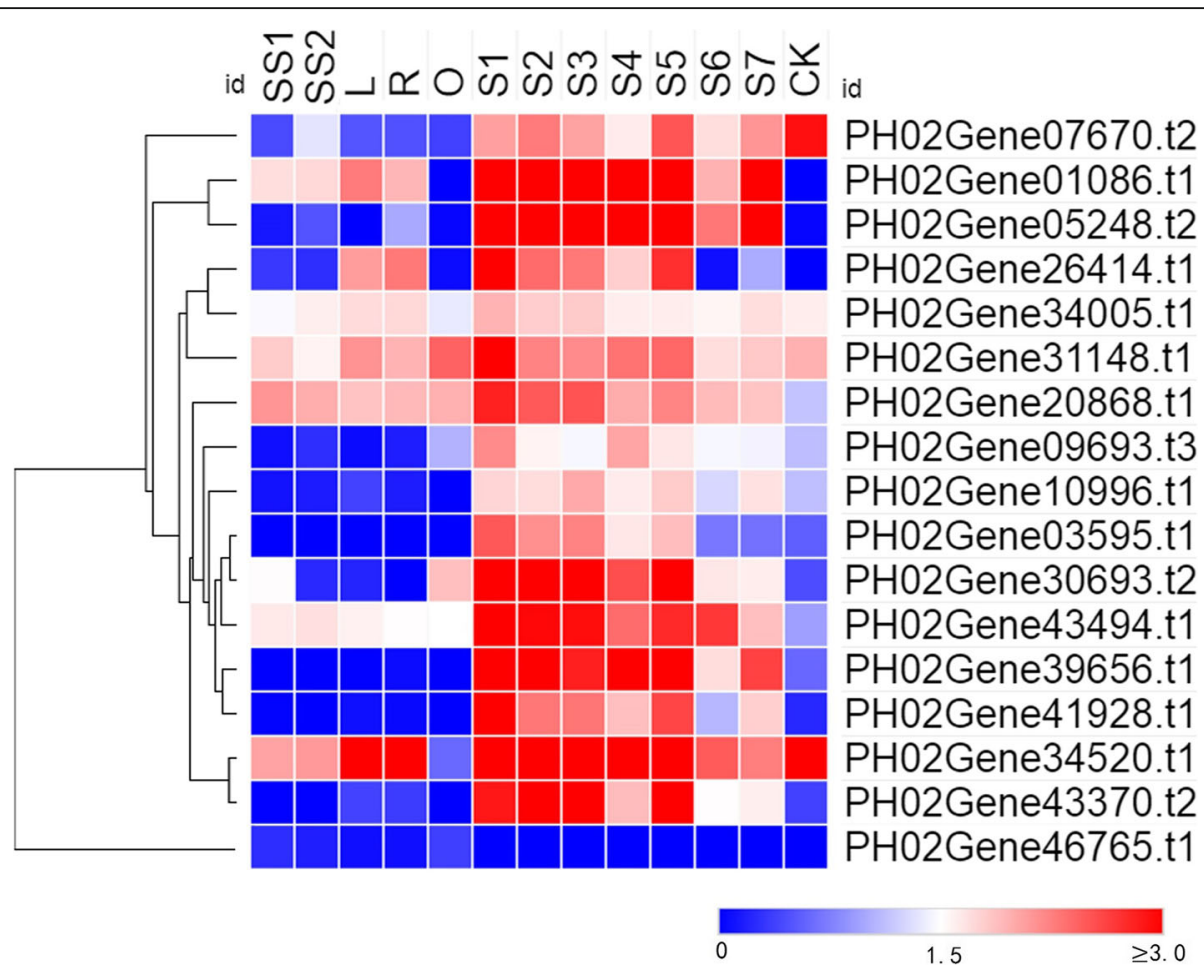

Fig. 5 Expression patterns of E2F/DP genes in different Moso bamboo tissues. The color scale represents log2-transformed fragments per kilobase of transcript per million mapped reads (FPKM) values. Blocks with colors indicate high accumulation levels (red) or low accumulation levels (blue). S1-S7 and CK represent winter bamboo shoots; 50-, 100-, 300-, 600-, 900-, and 1200-cm height bamboo shoots; and one-year-old mature culms, respectively. SS1, SS2, L, R and O represent the $1.5-\mathrm{cm}$-tall seedling stem, $8-\mathrm{cm}$-tall height seedling stem, lateral bud, rhizome and outward rhizome, respectively

regions and $\mathrm{PCC}(\mathrm{R}>0.90$ or $\mathrm{R}<-0.90)$ screening, a total of 58 genes had a strong correlation with at least one PheE2F/DP gene (Fig. 6). In the coexpression network, the primary hub gene PH02Gene26414.t1, which had the highest connecting times, positively regulated the expression of PH02Gene35099.t1 (LSD1), PH02Gene44324.t1 (ATXR3), PH02Gene09249.t1 (HOP), etc. In addition, PH02Gene05248.t2 and PH02Gene01086.t1 had the second and third-highest connecting times. All three hub genes reached the highest accumulation level in winter bamboo shoots, and all of them were regulated by PH02Gene08546.t1 (MYB), PH02Gene20517.t1 (MYB) and PH02Gene25898.t2 (MYB). In addition, all three genes regulated the expression of cell cycle-associated genes, including PH02Gene32333.t1 (CDKF), PH02Gene29974.t1 (MRB1), PH02Gene36158.t1 (SPR2), and PH02Gene33706.t1 (MCM2) as well as DNA replication-associated genes, including PH02Gene32448.t3 (POLD3) and PH02Gene37503.t2 (POLA2). Furthermore, many genes involved in RNA processing or environmental stress response showed high correlations with many E2F/DP members. The in situ hybridization data showed that the mRNAs of the hub genes PHO2Gene01086.t1 and PHO2Ge$n e 26414 . t 1$ were highly expressed in ground tissues of winter bamboo shoots but showed low expression levels in vascular bundles (Fig. 7).
In Arabidopsis, E2F can interact with DP to activate the cell cycle and cell proliferation-associated gene expression [8]. Thus, the yeast two-hybrid experiment was used to examine whether an E2F-DP complex also occurs in Moso bamboo. The three hub genes, PH02Gene34520.t1 (E2F), PH02Gene26414.t1 (DP), and PH02Gene34005.t1 (DP), which showed the highest accumulation level in winter bamboo shoots, were selected and further tested. The combinations of pGBKT7-PH02Gene26414.t1 + pGADT7PH02Gene34520.t1 and pGBKT7-PH02Gene34005.t1 + pGADT7-PH02Gene34520.t1 were co-transformed into yeast strain $\mathrm{AH} 109$. The $\mathrm{Y} 2 \mathrm{H}$ results showed that the positive control, negative control, and both test transformants grew well on SD/-Leu/-Trp medium (Fig. 8). The positive control and the experimental group turned blue when the transformants had grown on SD/-Trp/-Leu/-Ade/-His/ $\mathrm{X}-\alpha-\mathrm{Gal}$ media for 5 days. By contrast, the negative control could not grow on that nutritional selection media, which contained $\mathrm{X}-\alpha-\mathrm{Gal}$. These experimental results indicated that PheE2Fs can interact with PheDPs in Moso bamboo.

\section{Expression patterns of PheE2F/DPs under abiotic stress treatment and diurnal rhythms}

The regulatory analysis revealed that most PheE2F/DP promoters harbored various types of hormones and light 


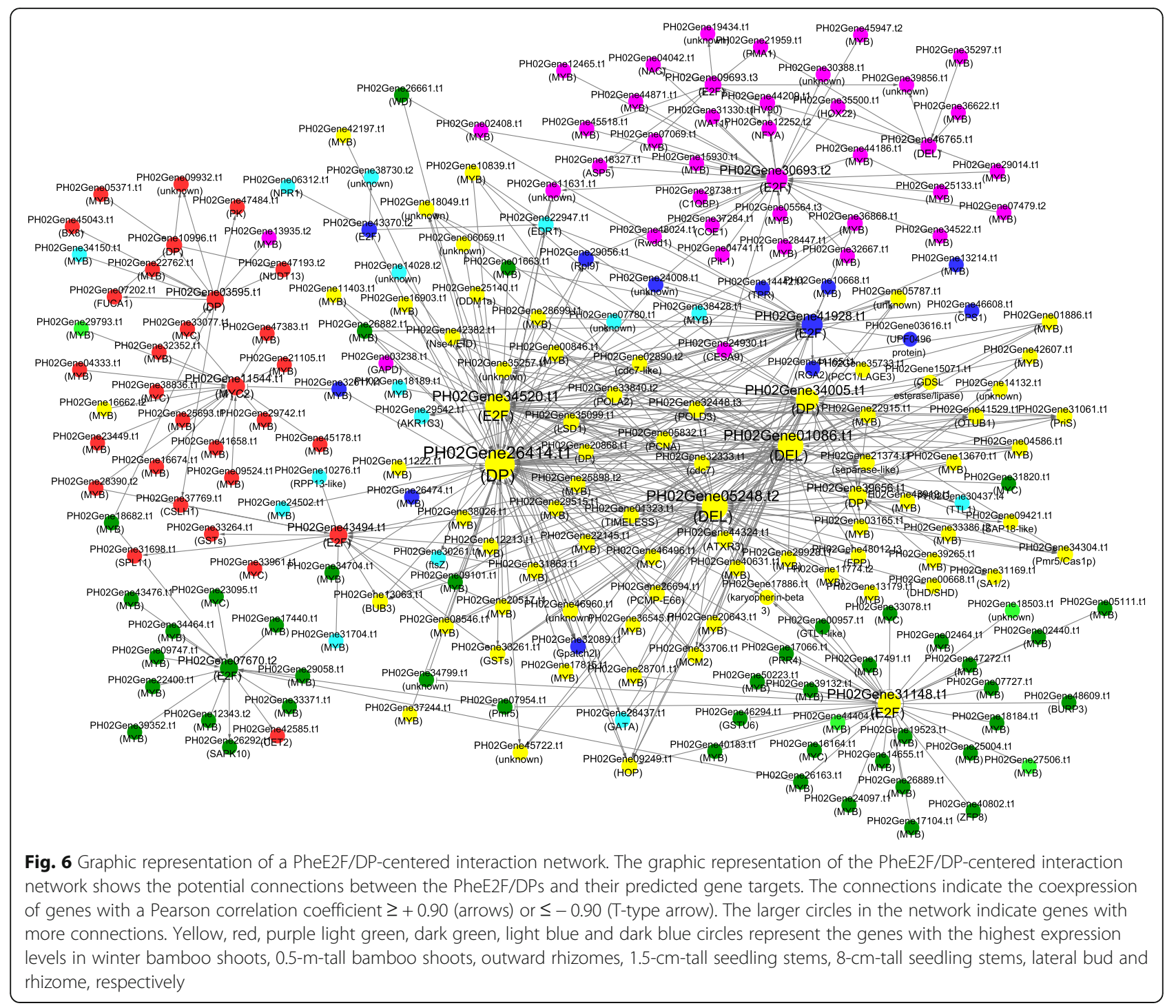

response elements. Hormone response elements, especially those of stress-related hormones such as abscisic acid, methyl limonate and salicylic acid, indicate that these genes might be induced or repressed by abiotic stress. To understand the expression changes of PheE2F/ $D P s$ under abiotic stress, the Moso bamboo seedlings were treated with drought (PEG) and salt treatments (Fig. 9). Under drought stress, the expression levels of PH02Gene07950.t1, PH02Gene07670.t2, PH02Gene09693.t3, PH02Gene10996.t1, PH02Gene26414.t1, PH02Gene34005.t1, PH02Gene01086.t1, PH02Gene05248.t2, and PHO2Gene18408.t2 were downregulated, while those of PH02Gene34520.t1, PH02Gene34267.t1, and PH02Gene42115.t1 were upregulated. The expression of PH02Gene03595.t1, PH02Gene20868.t1, and PH02Gene43494.t1 peaked at $12 \mathrm{~h}, 1 \mathrm{~h}$, and $1 \mathrm{~h}$, respectively. Under salt stress, PH02Gene30693.t2,
PH02Gene31148.t1, PH02Gene34520.t1, PH02Gene43370.t2, PH02Gene34267.t1, PH02Gene26414.t1, and PH02Gene34267.t1 showed upregulated expression trends, and the other PheE2F/DPs showed no significant differences in expression or downregulated expression trends.

To understand the transcriptional changes in PheE2F/ $D P s$ during diurnal cycles, we investigated the expression profiles of the bamboo shoots at $4 \mathrm{~h}$ intervals over a $24 \mathrm{~h}$ period (Fig. 10). The qRT-PCR analysis indicated that most PheE2FDPs were regulated by diurnal cycles. The transcription levels of PHO2Gene07950.t1, PHO2Gene31148.t3, PH02Gene03595.t1, and PH02Gene26414.t1 peaked at 15:00, 09:00, 06:00, and 12:00, respectively. The transcriptional levels of PH02Gene34520.t1, PH02Gene18408.t2 and PH02Gene42115.t1 remained at their maximum values from 4:00 to 19:00 (daytime). 


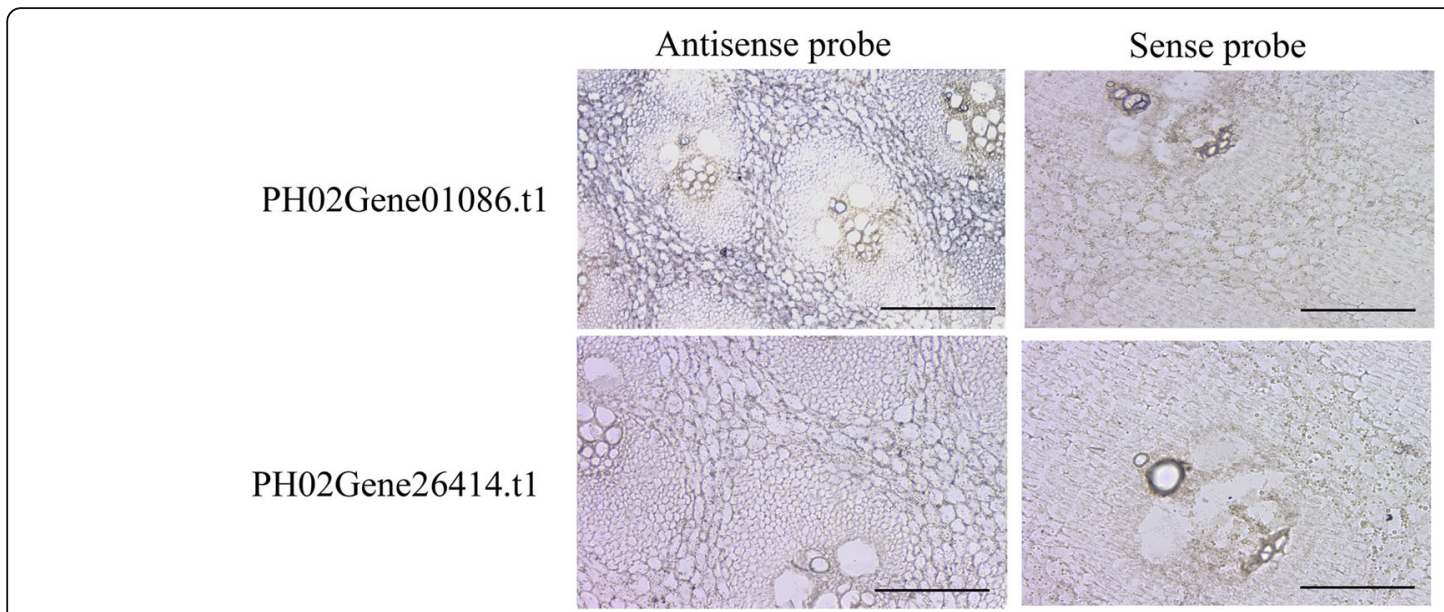

Fig. 7 In situ hybridization of PH02Gene01086.t1 and PH02Gene20868.t1 in winter Moso bamboo shoots

\section{Discussion}

Features of the Moso bamboo E2F/DP gene family

E2F/DP controls the spatial and temporal expression of genes that are essential for multiple biological processes during the cell cycle, and their transcription level determined the cell proliferation [7]. In a genome-wide screening, 23 E2F/DP genes harboring conserved domains were identified in Moso bamboo, which was much higher than the numbers identified in rice (8), Arabidopsis (7), Zea mays (19), wheat (18) and Brachypodium (11) [15-17]. The Moso bamboo genome size (2021 Mb) is comparable to that of Zea mays (2300 Mb) [18], much smaller than that of wheat (Triticeae) (17 Gb) [19], and much larger than that of Brachypodium (300 Mb), Arabidopsis (164 Mb) and Oryza sativa (441 Mb) [20-22]. Rather than the genome size, the much higher number of PheE2F/DPs in Moso bamboo indicated that the abundance of PheE2F/DPs may be associated with genome duplication events. Previous reports suggested that
Moso bamboo was tetraploid in origin, it went through a long evolutionary progression from tetraploidy to diploidy, and it carried two duplicates, similar to Oryza sativa gene model sets $[13,23]$. A similar phenomenon was consistently observed in the E2F/DP family. Eight PheE2F/DP paralogous gene pairs located in synteny blocks were identified in the Moso bamboo genome. In addition, most divergence times of the PheE2F/DP paralogous pairs in Moso bamboo occurred 7-12 mya, consistent with the recent whole-genome duplication time. These results suggested that the whole-genome duplication event was a major driving force of PheE2F/DP family expansion.

\section{PheE2F/DP proteins are involved in Moso bamboo shoot development}

Recently, several studies suggested that PheE2F/DP participated in many important biological processes in plants, such as leaf growth $[24,25]$, root growth [8], the

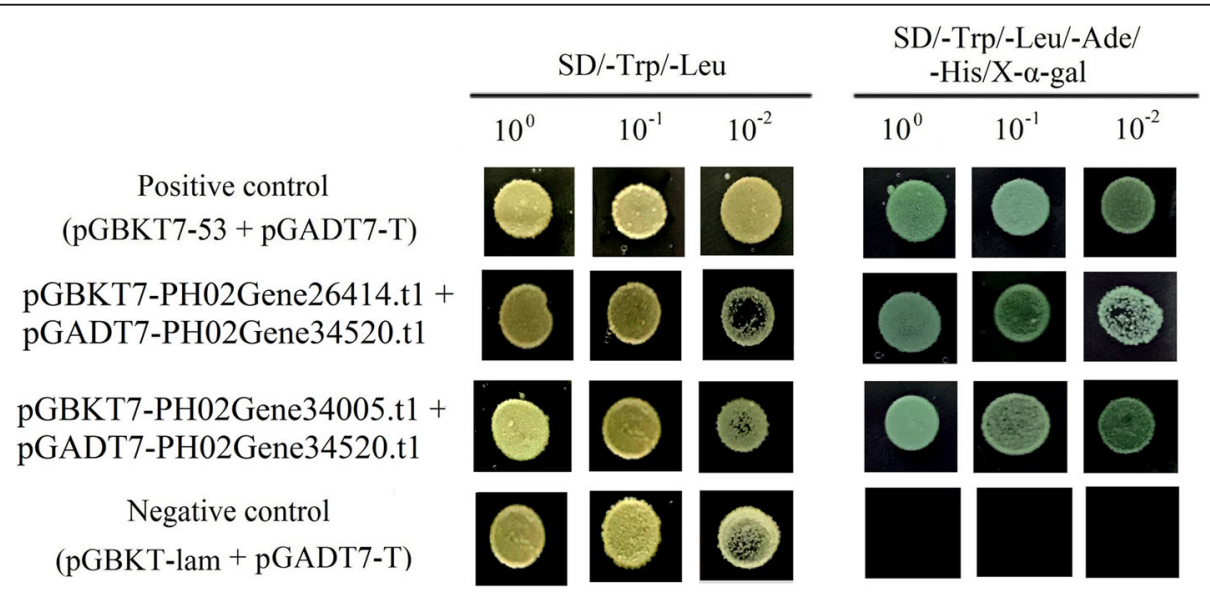

Fig. 8 The interaction between PheE2Fs and PheDPs. Positive control, co-transformation with pGBKT7-53 and pGADT7-T; negative control, cotransformation with pGBKT7-Lam and PGADT7-T 


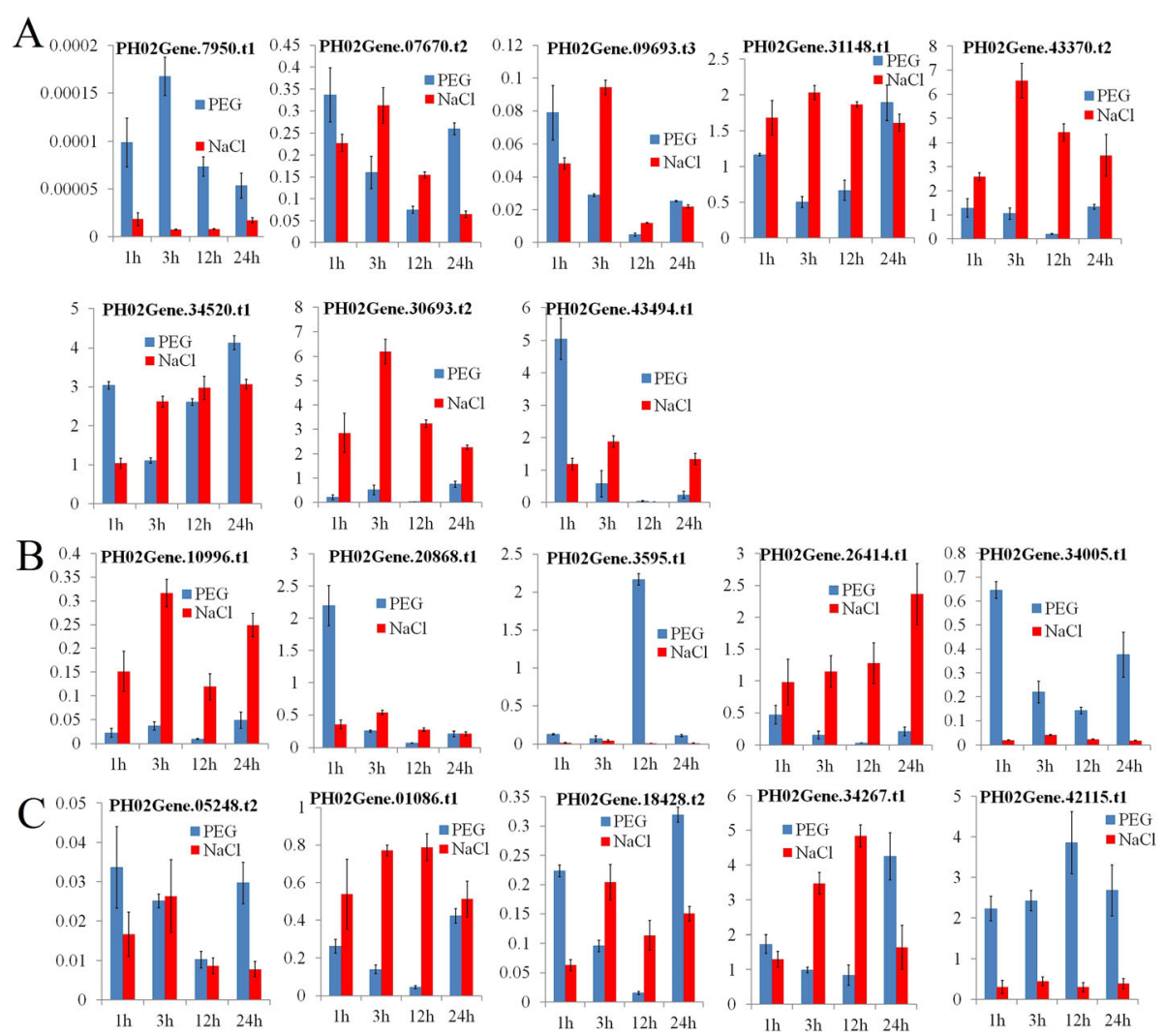

Fig. 9 Expression patterns of PheE2F/DP genes after being treated for 1, 3, 12, and $24 \mathrm{~h}$ under drought stress (blue column) or salinity stress (red column). The Y-axis indicates the relative expression level. The 1, 3, 6, 12, and 24 labels (x-axis) indicate the treatment time (hours) under the corresponding abiotic stresses. The qRT-PCR data were normalized using the TIP41 gene. For each time point, seedlings irrigated with tap water were regarded as a reference. a Relative expressions of E2F group genes under abiotic stresses. $\mathbf{b}$ Relative expressions of DP group genes under abiotic stresses. c Relative expressions of DEL group genes under abiotic stresses

maintenance of genome integrity and viability [26], and environmental stress response [27]. The expression analysis showed that more than half of the PheE2F/DPs, including five E2Fs and four DPS, showed the highest expression in winter bamboo shoots (Fig. 5). The screening of the binding sites at the promoter regions suggested that a large number of genes that participated in the cell cycle were regulated by the PheE2F/DP family, such as CDKF, MRB1, SPR2 and MCM2 (Fig. 6) [28], and most of these downstream genes also showed high expression levels during the winter and the early growth period. Previous studies suggested that intercalary meristem cells grew and divided continuously and vigorously during the winter and early growth periods (S1-S5), especially during the winter, but the intercalary meristem growth activity decreased during the late period, and the continuous growth of the bamboo shoot was substituted by cell elongation [2]. In Arabidopsis, E2Fa and E2Fb interact with $\mathrm{DPa}$ to regulate downstream gene expression [8]. In this study, we used the $\mathrm{Y} 2 \mathrm{H}$ assay to detect the interaction between E2Fs and DPs, and three hub genes which showed the highest accumulation levels in winter bamboo shoots were selected and further examined. The experiment results showed that the E2F-DP complex also occurs in Moso bamboo, which provides favorable evidence that the PheE2F-PheDP complex may play important roles in winter bamboo shoot growth. Thus, we concluded that the high abundance of E2F/DP genes during the winter and early growth period was essential for E2F-DP complex formation and that the E2FDP complex vigorously promoted meristem cell growth by activating the expression of the cell cycle genes.

A coexpression network analysis suggested that PH02Gene2641.t1, PH02Gene05248.t2 and PH02Gene01086.t1 were regulated by PH02Gene08546.t1 (MYB), PH02Gene20517.t1 (MYB) and PH02Gene25898.t2 (MYB). In addition, several MYB genes, such as PHO2Gene26882.t1 and PH02Gene09101.t1, repressed the expression of $E 2 F / D P$ genes (Fig. 6). In tobacco, the MybA1 and MybA2 mRNAs fluctuated and peaked at the mitotic period and regulated the expression of the cell cycle genes. In the transient expression assays, MybA1 and MybA2 activated the MSA-containing (M-specific activator) promoters, whereas MybB repressed these promoters [29]. In 


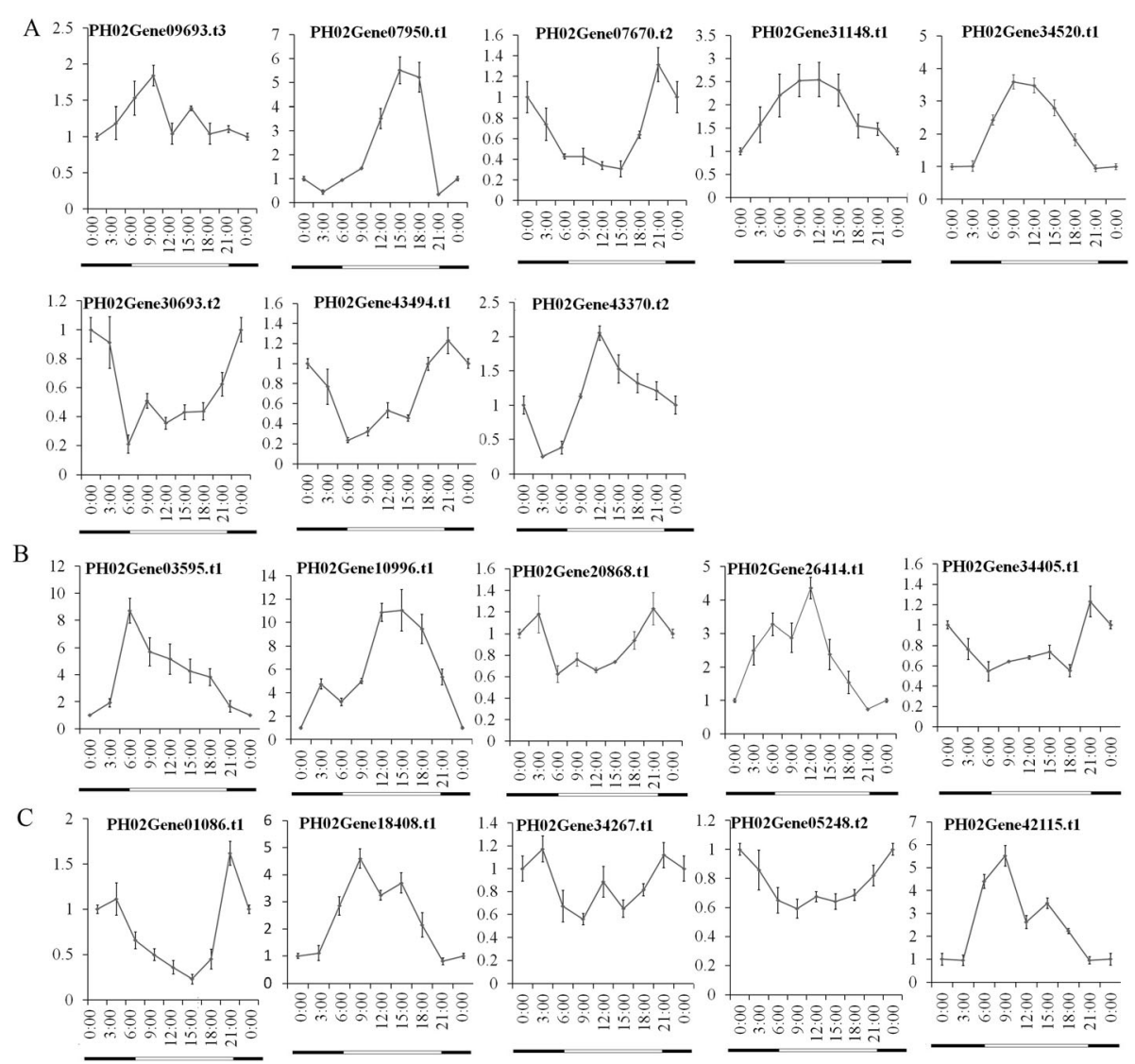

Fig. 10 Diurnal changes in the relative transcript abundance of PheE2F/DP genes analyzed by qRT-PCR. The Y-axis indicates the relative expression level and the $\mathrm{X}$-axis represents different time points. The white and black bars below the graph represent day and night, respectively. Bamboo tissues were collected every $3 \mathrm{~h}$ over a 48-h period from one-month-old bamboo seedlings. The expression of genes at each time point was averaged using two days of data. TIP41 was used to normalize the expression data. a Relative expressions of E2F group genes under abiotic stresses. $\mathbf{b}$ Relative expressions of DP group genes under abiotic stresses. $\mathbf{c}$ Relative expressions of DEL group genes under abiotic stresses

the current study, all 23 promoters contained MYB binding sites, and most $E 2 F / D P$ genes showed positive or negative correlations with MYBs. Thus, under the control of the MYBs, the E2F/DP genes played critical roles in regulating the expression of the cell cycle genes during Moso bamboo shoot growth and development.

\section{PheE2F/DP proteins are involved in stress and light responses}

Based on the regulatory element analysis, a large number of light response sites were identified in the PheE2F/DP promoters. The expression analysis based on qRT-PCR also confirmed that most PheE2F/DPs were photoresponse genes. The transcriptional levels of $\mathrm{PHO} \mathrm{Ge}^{-}$ ne18408.t2 and PH02Gene42115.t1 remained at the maximum value during the daytime in the Moso bamboo shoots, while PHO2Gene30693.t2 and PHO2Gene07670.t2 were highly expressed at night (Fig. 10). In Arabidopsis, light alters the balance of E2FC and E2FB, with opposing functions in the regulation of cell proliferation. Light increases the expression of E2FB protein levels and further induces the expression of cell cycle genes [30]. Thus, we speculated that PH02Gene30693.t2 and PH02Gene07670.t2, which showed high accumulation levels at night, might act as negative regulators of the cell cycle and cell proliferation, and their high abundance at night might repress the expression of cell cycle genes. During the daytime, light increased the abundance of PH02Gene18408.t2 and PH02Gene42115.t1 and activated their expression, further accelerating lightmediated Moso bamboo shoot growth and development.

The identification of regulatory elements in the PheE2F/DP promoters indicated that most members harbored abscisic acid, MeJA and salicylic acid regulatory elements, indicating that the expression of these genes might be stimulated by some environmental cues. The qRT-PCR analysis also provided evidence that several PheE2F/DP genes were induced by abiotic stress (Fig. 9). Coexpression network analysis suggested that many environmental stress response genes were regulated by E2F/DPs. PH02Gene44788.t1 (AtRGGA), which showed a high correlation with PH02Gene41928.t1 (E2F/ 
$D P$ ) and PH02Gene26414.t1 (E2F/DP), is involved in stress responsiveness. In Arabidopsis, AtRGGA is induced by salt and osmotic stress, and atrgga mutants exhibit increased sensitivity to abiotic stress [31]. In addition, PH02Gene25752.t1 (an ABA-responsive gene) and PH02Gene37465.t1 (a plant viral response gene) were also regulated by E2F/DPs in the network [32]. The high accumulation level of several PheE2F/DPs under abiotic stress might be essential for plant resistance to numerous adverse environments.

The regulation of the cell cycle might be subject to interference by drought and salt stress at the transcriptional level through repression of E2F expression. However, there is a great deal of new evidence that suggests that drought and salt stress can stimulate the expression of cell cycle genes. For the Medicago truncatula E2Fb gene, an upregulation trend was reported under high salt concentration treatment [3, 27]. In addition, many studies have also revealed the relationship between the accumulation level of cell cycle regulators and salt stress. The expression of Oryza CDKC1, which is involved in cell proliferation and differentiation, was triggered by $\mathrm{NaCl}$ treatment through the ABA signaling pathway [33]. In Arabidopsis, CDC2aAt, a cyclin-dependent kinase-coding gene and two mitotic cyclin genes, namely, Arath;CycB1;1 and Arath;CycA2;1, were monitored during salt stress [34]. In mammals, many E2F members act as key factors in the DNA damagedependent expression regulation of cell cycle genes [35]. Many PheE2F/DPs showed high accumulation levels under abiotic stress, which may facilitate bamboo growth under harsh environments. Collectively, PheE2F/DPs participated in different biological processes in Moso bamboo.

\section{Conclusion}

In conclusion, a total of 23 PheE2F/DPs were identified in the Moso bamboo genome, including nine E2F, six DP, and eight DEL genes. The cis-acting elements involved in the photoperiodic response, hormone signaling, meristem growth, and many MYB and MYC2 binding sites were present in the PheE2F/ $D P$ promoters. Recent whole-genome duplication played important roles in the E2F/DP family expansion. Transcriptome sequencing and in situ hybridization analysis revealed that $E 2 F / D P s$ play important roles in bamboo shoot growth, especially during the winter period. Expression profiles derived from $\mathrm{qRT}-\mathrm{PCR}$ indicated that $E 2 F / D P$ expression is stimulated by various forms of abiotic stress and diurnal cycles. These results provide comprehensive insights into the Moso bamboo E2F/DP gene family and lay a solid foundation for further functional verification of the PheE2F/DP family.

\section{Methods}

\section{Database searches}

The E2F/DP sequences of Arabidopsis thaliana (Arabidopsis), Oryza sativa (rice), and Brachypodium distachyon (Brachypodium) were downloaded from The Arabidopsis Information Resource (TAIR) [36], the rice genome annotation project [37], and the Brachypodium distachyon genome database [38], respectively. The published $A t E 2 F / D P$ and $O s E 2 F / D P$ sequences were then used as queries in BLASTP searches against the local bamboo protein database using the default parameters. The sequences were considered as predicted E2F/DPs if they had E-values of $\leq-10$. Lastly, the protein families database (Pfam) was further used to confirm each candidate E2F/DP sequence.

\section{Plant materials}

Moso bamboo shoots as well as other culm samples were collected by Long $\mathrm{Li}$ in Guangde County (E119 $\left.41^{\prime} ; \mathrm{N} 30^{\circ} 89^{\prime}\right)$, Anhui Province, from January to August 2018 (Additional file 1: Fig. S2). The formal identification of these culm samples were performed by $\mathrm{Li}$ et al. [2]. The bamboo samples were frozen in liquid nitrogen and stored at $-80^{\circ} \mathrm{C}$ prior to RNA extraction. Permission to collect bamboo shoots and other culm samples for the experiments was obtained from the forestry bureau of Guangde County. Moso bamboo seeds were collected by Long $\mathrm{Li}$ in Dajing County, Guilin (E110 179'; N25 049') in Guangxi Zhuang Autonomous Region from July to September, 2018 (Additional file 1: Fig. S2). The necessary field work permits were obtained from the Guilin Forestry Bureau. The formal identification of these seeds was performed by Gao et al. [39]. In addition, the sample collection work did not affect the ecology and did not involve protected species.

The circadian rhythm expression experiment was conducted in artificial climate chambers with supplemental light from 4:00 to 19:00. Bamboo tissues were collected by Long Li every $3 \mathrm{~h}$ during a 48-h period from onemonth-old bamboo seedlings. Five individuals that showed similar growth patterns were pooled at each time point and three biological repeats were conducted for each sample.

For the abiotic stress treatment, bamboo seedlings were grown in artificial climate chambers under longday conditions ( $15 \mathrm{~h}$ of light $/ 9 \mathrm{~h}$ of dark). A drought stress test was conducted by irrigating the two-monthold seedlings with 18\% PEG6000 medium. A salt stress test was conducted by irrigating the two-month-old seedlings with medium containing $250 \mathrm{mM} \mathrm{NaCl}$. The leaves were harvested by Long $\mathrm{Li}$ at $1,3,12$ and $24 \mathrm{~h}$ after abiotic stress treatment. Seedlings irrigated with tap water were used as the control (CK). The plant samples were frozen in liquid nitrogen and stored at $-80^{\circ} \mathrm{C}$ 
prior to use. Five individuals that showed similar growth patterns were pooled at each time point and three biological repeats were employed for each sample. The voucher specimens were deposited in the herbarium at the International Center for Bamboo and Rattan, Beijing, catalog numbers ICBR-B-18101 (bamboo shoot), ICBRB-18201 (rhizome), ICBR-B-18301(outward-rhizome), ICBR-B-18401 (seedling stem), ICBR-B-18501 (seed), ICBR-B-18601 (seedling leaf).

\section{Gene structure, conserved motif and regulatory element analysis}

The Gene Structure Display Server program (GSDS) was used to investigate the gene structure of E2F/DP based on the GFF annotation file with the default parameters [40]. The conserved motifs in the identified E2F/DP sequences were identified using the Multiple EM for Motif Elicitation program (MEME) [41]. The MEME was run locally using the following parameters: number of repetitions - any, maximum number of motifs - 8 , and the optimum motif widths were constrained to between 25 and 200 residues.

The transcription start sites (mRNA start sites) were designated as +1 and obtained by GFF file as downloaded from the GigaDB, the new version of the Moso bamboo genome annotation project [23]. The regulatory elements in the promoter region of PheE2F/DPs as well as the other 51,050 Moso bamboo genes (from $-2000 \mathrm{bp}$ to $+1 \mathrm{bp}$ ) were analyzed using the PlantCARE online program $[14,42]$. A GO annotation of all the Moso bamboo genes was performed using TBtools based on the BLASTX method. BLASTX results with an e-value cutoff of $1 \mathrm{e}^{-6}$ and a minimum similarity of $55 \%$ were annotated with GO terms. The promoters of genes that contained E2F/ DP binding sites were further used for Gene Ontology (GO) enrichment analysis via TBtools [43].

\section{Collinearity analysis}

To investigate the synteny of blocks containing E2F/DP genes, homology data derived from protein-protein comparisons made using BLASTN, with an E-value cutoff of $1 \mathrm{e}-6$, and the other parameters were the defaults or recommended ones. The BLASTP result was analyzed using MCScanX to find the collinear regions between PheE2F/ $D P$ genes as well as other synteny blocks across the whole genome. The minimum block size was 5 and the other parameters were the default or recommended ones. The synteny blocks were extracted to draw a collinearity map within PheE2F/DPs using TBtools software.

Estimation of the divergence time in paralogous pairs The Ka (nonsynonymous substitution), Ks (synonymous substitution) and the $\mathrm{Ka} / \mathrm{Ks}$ ratio between the paralogous pairs were calculated using DNA Sequence Polymorphism software (DnaSP). The formula $\mathrm{T}=\mathrm{Ks} / 2 \lambda$ was used to estimate the divergence time of the duplication event, with a divergence rate $\lambda=6.5 \times 10^{-9}$ in monocotyledons and $1.5 \times 10^{-8}$ in dicotyledons [44].

\section{Multiple sequence alignment and phylogenetic tree construction}

The ClustalW program was executed for multiple sequence alignments using the full-length E2F/DP amino acids with the default settings. The parameters used to run the CLUSTALW program were as follows: Scoring matrix for amino acid sequences: BLOSUM62, gap open: 10, gap extension: 0.2, iteration: none. The Maximum Likelihood tree was built using the IQ-Tree software with the best fit tree model, and the JTT + R3 was found to be the best model according to the Bayesian Information Criterion. The bootstrap method was applied in phylogeny test with 1000 replications. After that, the results were imported to FigTree v1.4.2 software for processing.

\section{RNA extraction, reverse transcription and qRT-PCR analysis}

The total RNA from each sample was isolated using the TRIzol reagent (Invitrogen, USA). First-strand cDNA was synthesized with $2 \mu \mathrm{g}$ RNA using a PrimeScriptTM RT Reagent Kit (TaKaRa, Japan) according to the manufacturer's instructions. qRT-PCR experiments were performed using SYBR Green chemistry (Roche, Mannheim, Germany) on a Light Cycler 480 instrument (Roche, Rotreuz, Switzerland) according to the manufacturer's directions. The Primer 3 online program was used for gene-specific primer design (Additional file 1: Table S2) [45]. Three biological replicates and three technical replicates were employed in each experiment. The TIP41 was selected as an internal control [46]. The final relative expression levels were calculated using the $2^{-\Delta \Delta} \mathrm{Ct}$ method.

\section{Transcriptome data analysis}

The transcriptome data for PheE2F/DPs from the developing rhizome-root systems and bamboo shoots were previously generated and processed (PRJNA354950, PRJNA604634, PRJNA706151) [2, 12]. These transcriptome data were generated from different types of growing culms, including a 1.5 -cm-tall seedling stem (SS1), 8-cm-tall seedling stem (SS2), lateral bud (L), rhizome (R), outward rhizome $(\mathrm{O})$, and bamboo shoots at different growth stages (winter bamboo shoots and 50-, 100-, 300-, 600-, 900- and 1200-cm-tall shoots, designated S1S7, respectively (Additional file 1: Fig. S2). Following leaf expansion, the culms were labeled as CK. The four growing culms (S1, O, SS1 and R) were sampled when 
the culm length reached one-tenth of the final length. The expression abundance of PheE2F/DPs was measured as fragments per kilobase of exon model per million mapped reads (FPKM). The heatmap was generated using R.

\section{Regulation network of PheE2Fs}

The transcription factor-target gene (TF-TG) interactions were predicted based on the regulatory elements in the promoters of the target genes. The gene expression correlation was calculated by Pearson correlation coefficient (PCC) using FPKM through the R program. Only the transcription factor (TF) and target relationships that displayed PCC values of $\geq+0.90$ or $<-0.90$ were retained for coexpression network construction. The network was ultimately visualized using Cytoscape 3.7.0 software.

\section{In situ hybridization}

Bamboo shoot tissues were fixed in $4 \%$ paraformaldehyde for one day at $4{ }^{\circ} \mathrm{C}$ and then hybridized as described previously [2]. The specific probes of PH02Gene01086.t1 and PH02Gene26414.t1 were amplified by PCR using gene-specific primers with T7 and SP6 RNA polymerase-binding sites. The images were captured using an Olympus Nikon E600.

\section{Yeast two-hybrid assay}

To test whether E2F can interact with DP in Moso bamboo, the Matchmaker GAL4 two-hybrid system (Clontech, Palo Alto, CA) was applied. Based on the in-fusion cloning method (Clontech, Palo Alto, CA), the full length of the PH02Gene26414.t1 and PH02Gene34005.t1 cDNAs were independently cloned into pGBKT7, and the full length PH02Gene34520.t1 cDNAs were cloned into pGADT7 (Additional file 1: Table S3). Next, the combinations of pGBKT7-PH02Gene26414.t1 + pGADT7-PH02Gene34520.t1 and pGBKT7-PH02Gene34005.t1 + pGADT7PH02Gene34520.t1 were co-transformed into the AH109 yeast strain. The transformed yeasts were cultured on $\mathrm{SD} /$ -Trp/-Leu for 4 days and then transferred to SD/-Trp/ -Leu/-Ade/-His/X- $\alpha$-Gal plates for 5 days. The cotransformants containing pGBKT7-53 + pGADT7-T and pGBKT7-Lam + pGADT7-T were selected as the positive control and negative control, respectively.

\footnotetext{
Abbreviations

ABA: Abscisic acid; ABRE: Abscisic acid responsiveness; CDC: Cell division cycle; CDKC: Cyclin dependent kinase C; CREs: Cis-regulatory elements; DNnaSP: DNA Sequence Polymorphism; FPKM: Fragments per kilobase of exon model per million mapped reads; GSDS: Gene Structure Display Server program; GO: Gene ontology; Ka: Nonsynonymous substitution; Ks: Synonymous substitution; MEGA: Molecular evolution genetics analysis; MeJA : Methyl jasmonate; MEME: Multiple em for motif elicitation; MYA: Million years ago; NJ: Neighbor-joining; PCC: Pearson correlation coefficient; Pfam: Protein families database; PEG: Polyethylene glycol; qRT-
}

PCR: Quantitative real-time PCR; TAIR: Arabidopsis Information Resource; TFTG: Transcription factor-target gene; Y2H: Yeast two hybrid

\section{Supplementary Information}

The online version contains supplementary material available at https://doi. org/10.1186/s12870-021-02924-8.

Additional file 1: Figure S1. Sequence logo of the different motifs identified in the PheE2F proteins. Figure S2. Moso bamboo tissues used for expression analysis. Table S1. Estimated divergence period of E2F/DP gene pairs in four species. Table S2. The primer sequences used for qRTPCR. Table S3. The primer sequences used for gene cloning

\section{Acknowledgements}

Not applicable.

\section{Authors' contributions}

L.L. performed bioinformatics analyses, sample collection, experiments and drafted the manuscript. Q.S. and Z.L. helped in bioinformatics analyses and physiological experiments. J.G. designed the experiments and conceived the project, provided overall supervision of the study and revised the manuscript. All authors have read and approved the final manuscript.

\section{Funding}

This work was supported by the National Key Research and Development Program of China (2018YFD0600101); Natural Science Foundation of Shaanxi Province (Grant No. 2019JQ-402), supported by the Shannxi Provincial Department of Science and Technology; the Fundamental Research Funds of ICBR (Grant No. 1632018006), supported by the International Bamboo and rattan Center; the Scientific Startup foundation for Doctor of Northwest A\&F University (grant No. Z109021715), a project funded by the Northwest Agriculture \& Forestry University. Each of the funding bodies granted the funds based on a research proposal, and the funders played no role in study design, data analysis, and manuscript preparation.

\section{Availability of data and materials}

All the RNA-Seq raw data are available at NCBI under accession number PRJNA354950 (https://www.ncbi.nlm.nih.gov/bioproject/?term=PRJNA35495 0), PRJNA604634 (https://www.ncbi.nlm.nih.gov/bioproject/?term=prjna6 04634), PRJNA706151 (https://www.ncbi.nlm.nih.gov/bioproject/?term= PRJNA706151).

\section{Declarations}

Ethics approval and consent to participate

The permission of bamboo shoot and other culm samples collection for the experiments was obtained from forestry bureau of Jingxian County. The permission of bamboo seeds collection for the experiments was obtained from Guilin Forestry Bureau.

\section{Consent for publication}

Not applicable.

\section{Competing interests}

All the authors have declared no conflict of interest.

\section{Author details \\ ${ }^{1}$ College of Forestry, Northwest A\&F University, Yangling 712100, Shaanxi, China. ${ }^{2}$ College of Landscape Architecture and Art, Northwest A\&F University, Yangling 712100, Shaanxi, China. ${ }^{3}$ International Center for Bamboo and Rattan, Key Laboratory of Bamboo and Rattan Science and Technology, State Forestry Administration, Beijing, China.}

Received: 22 July 2020 Accepted: 11 March 2021

Published online: 29 March 2021

References

1. Wei Q, Guo L, Jiao C, Ding YL, Gao S, Guo L, Chen M, Hu P, Xia SJ, Ren GD, Fei ZJ. Characterization of the developmental dynamics of the elongation of 
a bamboo internode during the fast growth stage. Tree Physiol. 2019;39(7): 1201-14. https://doi.org/10.1093/treephys/tpz063.

2. Li L, Cheng ZC, Ma YJ, Bai QS, Li XY, Cao ZH, Wu ZN, Gao J. The association of hormone signaling, transcription and anatomy during shoot growth in Moso bamboo. Plant Biotechnol J. 2018;16(1):72-85. https://doi.org/10.1111/ pbi.12750.

3. Ma TY, Li ZW, Zhang SY, Liang GT, Guo J. Identification and expression analysis of the E2F/DP genes under salt stress in Medicago truncatula. Genes Genom. 2014;36(6):819-28. https://doi.org/10.1007/s13258-014-0218-5.

4. Ramirez-Parra E, Lopez-Matas MA, Frundt C, Gutierrez C. Role of an atypical E2F transcription factor in the control of Arabidopsis cell growth and differentiation. Plant Cell. 2004;16(9):2350-63. https://doi.org/10.1105/tpc.1 04.023978.

5. del Pozo JC, Boniotti MB, Gutierrez C. Arabidopsis E2FC functions in cell division and is degraded by the ubiquitin-SCFAtSKP2 pathway in response to light. Plant Cell. 2002;14(12):3057-71. https://doi.org/10.1 105/tpc.006791

6. del Pozo JC, Diaz-Trivino S, Cisneros N, Gutierrez C. The balance between cell division and endoreplication depends on E2FC-DPB, transcription factors regulated by the ubiquitin-SCFSKP2A pathway in Arabidopsis. Plant Cell. 2006;18(9):2224-35. https://doi.org/10.1105/tpc.105.039651.

7. Magyar Z, Bogre L, Ito M. DREAMs make plant cells to cycle or to become quiescent. Curr Opin Plant Biol. 2016;34:100-6. https://doi.org/10.1016/j.pbi.2 016.10 .002$.

8. Sozzani R, Maggio C, Varotto S, Canova S, Bergonioux C, Albani D, Cella R. Interplay between Arabidopsis activating factors E2Fb and E2Fa in cell cycle progression and development. Plant Physiol. 2006;140(4):1355-66. https:// doi.org/10.1104/pp.106.077990.

9. Polager S, Ginsberg D. E2F - at the crossroads of life and death. Trends Cell Biol. 2008;18(11):528-35. https://doi.org/10.1016/j.tcb.2008.08.003.

10. Ramirez-Parra E, Friindt C, Gutierrez C. A genome-wide identification of E2Fregulated genes in Arabidopsis. Plant J. 2003;33(4):801-11. https://doi.org/1 0.1046/j.1365-313X.2003.01662.x.

11. Yan XY, Jiang T, Wang XQ, Qiu SH, Liu BB. Identification and related expression analysis of E2F/DP transcription factor gene family in Moso bamboo. Mol Plant Breed. 2018;16:6997-7003.

12. Wang T, Wang H, Cai D, Gao YB, Zhang HX, Wang YS, Lin CT, Ma LY, Gu LF. Comprehensive profiling of rhizome-associated alternative splicing and alternative polyadenylation in Moso bamboo (Phyllostachys edulis). Plant J. 2017;91(4):684-99. https://doi.org/10.1111/tpj.13597.

13. Peng ZH, Lu Y, Li LB, Zhao Q, Feng Q, Gao ZM, Lu HY, Hu T, Yao N, Li KY, et al. The draft genome of the fast-growing non-timber forest species Moso bamboo (Phyllostachys heterocycla). Nat Genet. 2013;45(4):456-61. https:// doi.org/10.1038/ng.2569.

14. Magali L, Patrice D, Gert T, Kathleen M, Yves M, Yves VP, Pierre R, Stephane R. PlantCARE, a database of plant cis-acting regulatory elements and a portal to tools for in silico analysis of promoter sequences. Nucleic Acids Res. 2002:30:325-7.

15. Luisa M, Barbara P, Rita C, Rebecca S, Catherine B, Rino C, Diego A. The E2F family of transcription factors from Arabidopsis thaliana. Novel and conserved components of the retinoblastoma/E2F pathway in plants. J Biol Chem. 2002;277(12):9911-9.

16. Cooper B, Hutchison D, Park S, Guimil S, Luginbühl P, Ellero C, Goff SA, Glazebrook J. Identification of rice (Oryza sativa) proteins linked to the cyclin-mediated regulation of the cell cycle. Plant Mol Biol. 2003;53(3):273-9.

17. Jin J, Tian F, Yang DC, Meng YQ, Kong L, Luo JC, Gao G. PlantTFDB 4.0: toward a central hub for transcription factors and regulatory interactions in plants. Nucleic Acids Res. 2017:45(D1):D1040-5.

18. Duvick J, Fu A, Muppirala U, Sabharval M, Wilkerson MD, Lawrence CJ, Lushbough C, Brendel V. PlantGDB: a resource for comparative plant genomics. Nuclear Acids Res. 2008;36:D959.

19. International Wheat Genome Sequencing Consortium. A chromosomebased draft sequence of the hexaploid bread wheat (Triticum aestivum) genome. Science 2014; 345: 1251788, A chromosome-based draft sequence of the hexaploid bread wheat (Triticum aestivum) genome, 6194, DOl: https://doi. org/10.1126/science.1251788

20. Burr B. Mapping and sequencing the rice genome. Plant Cell. 2002;14(3): 521-3. https://doi.org/10.1105/tpc.140310.

21. The International Brachypodium Initiative. Genome sequence analysis of the model grass Brachypodium distachyon: insights into grass genome evolution. Nature. 2010;463(7282):763-8. https://doi.org/10.1038/na ture08747.
22. Filichkin SA, Priest HD, Givan SA. Genome-wide mapping of alternative splicing in Arabidopsis thaliana. Genome Res. 2010;20(1):45-58. https://doi. org/10.1101/gr.093302.109.

23. Zhao HS, Gao ZH, Wang L, Wang JL, Wang SB, Fei B, Chen C, Shi C, Liu X, Zhang $\mathrm{H}$. Chromosome-level reference genome and alternative splicing atlas of Moso bamboo (Phyllostachys edulis). GigaScience. 2018;7:1-12.

24. Bénédicte D, Elena RP, Qi X, Chua NH, Crisanto G. Cell type-specific role of the retinoblastoma/E2F pathway during Arabidopsis leaf development. Plant Physiol. 2005;140(1):67-80

25. Erika Ö, Csaba P, Binish M, Aladár P, Tünde L. E2FB interacts with RETI NOBLASTOMA RELATED and regulates cell proliferation during leaf development. Plant Physiol. 2020;182(1):518-33.

26. Horvath BM, Kourova H, Nagy S, Nemeth E, Magyar Z, Papdi C, Ahmad Z, Sanchez-Perez GF, Perilli S, Blilou I. Arabidopsis RETINOBLASTOMA RELATED directly regulates DNA damage responses through functions beyond cell cycle control. EMBO J. 2017;36(9):1261-78. https://doi.org/10.15252/embj.201 694561.

27. Li D, Su Z, Dong J, Wang T. An expression database for roots of the model legume Medicago Truncatula under salt stress. BMC Genomics. 2009;10(1): 517. https://doi.org/10.1186/1471-2164-10-517.

28. Klaas VK, Vlieghe K, Florqui LH, Gerrit TSB, Wilhelm G, Yves VP, Dirk I, Lieven DV. Genome-wide identification of potential plant E2F target genes. Plant Physiol. 2005;139:316-28.

29. Ito M, Araki S, Matsunaga S, Itoh T, Nishihama R, Machida Y, Doonan JH, Watanabe A. G2/M-phase-specific transcription during the plant cell cycle is mediated by c-Myb-like transcription factors. Plant Cell. 2001;13(8):1891-905. https://doi.org/10.1105/tpc.010102.

30. Enrique LJ, Edyta D, Zoltán M, Safina K, Saul H, Sarah M, James AHM, Gerrit TS, László B, Hugh PS. Distinct light-initiated gene expression and cell cycle programs in the shoot apex and cotyledons of Arabidopsis. Plant Cell. 2008; 20(4):947-68.

31. Alfredo A, Giorgia B, Roberta N, Vincenzo A, Paola P, Dhinoth KB, Ida R, Massimiliano S, Antonietta L, Antonello C, Stefania G. The Arabidopsis AtRGGA RNA binding protein regulates tolerance to salt and drought stress. Plant Physiol. 2015;168(1):292-306.

32. Li T, Wu XY, Li H, Song JH, Liu JY. A dual-function transcription factor, AtYY1, is a novel negative regulator of the Arabidopsis ABA response network. Mol Plant. 2016;9(5):650-1. https://doi.org/10.1016/..molp.2016.02. 010.

33. Huang YW, Tsay WS, Chen CC, Lin CW, Huang HJ. Increased expression of the rice $\mathrm{C}$-type cyclin-dependent protein kinase gene, Orysa; $\mathrm{CDKC}$; 1 , in response to salt stress. Plant Physiol Bioch. 2008;46(1):71-81. https://doi. org/10.1016/j.plaphy.2007.10.013.

34. Sylvie B, Kristiina H, Brigitte C, Tom B, Marc VM, Dirk I, Nathalie V. Expression of cell cycle regulatory genes and morphological alterations in response to salt stress in Arabidopsis thaliana. Planta. 2000;211:632-40.

35. Mitxelena J, Apraiz A, Vallejo-Rodríguez J, García-Santisteban I, Fullaondo A, Alvarez-Fernández M, Malumbres M, Zubiaga AM. An E2F7-dependent transcriptional program modulates DNA damage repair and genomic stability. Nucleic Acids Res. 2018;9:9

36. Berardini TZ, Reiser L, Li D, Mezheritsky Y, Muller R, Strait E, Eva HL. The Arabidopsis information resource: making and mining the "gold standard" annotated reference plant genome. Genesis. 2015;53(8):474-85. https://doi. org/10.1002/dvg.22877.

37. Yoshihiro K, Bastide M, Hamilton JP, Kanamori H, McCombie WR, Ouyang S, Schwartz DC, Tanaka T, Wu J, Zhou S, Childs KL, Davidson RM, Lin H, QuesadaOcampo L, Vaillancourt B, Sakai H, Lee SS, Kim J, Numa H, Itoh T, Buell CR, Matsumoto T. Improvement of the Oryza sativa Nipponbare reference genome using next generation sequence and optical map data. Rice. 2013;6:4.

38. Vogel JP, Garvin DF, Mockler TC, Schmutz J, Rokhsar D. Genome sequencing and analysis of the model grass Brachypodium distachyon. Nature. 2010; 463(7282):763-8.

39. Gao J, Zhag Y, Zhang CL, Qi FY, Li XP, Mu SH, Peng ZH. Characterization of the floral Transcriptome of Moso bamboo (Phyllostachys edulis) at different flowering developmental stages by transcriptome sequencing and RNA-seq analysis. PLoS One. 2013;9(6):e98910.

40. Hu B, Jin JP, Guo AY, Zhang H, Luo CJ, Gao G. GSDS 2.0: an upgraded gene feature visualization server. Bioinformatics. 2015;31(8):1296-7. https://doi. org/10.1093/bioinformatics/btu817.

41. Bailey TL, Boden M, Buske FA, Frith M, Grant CE, Clementi L, Ren J, Li WW, Noble WS. MEME SUITE: tools for motif discovery and searching. Nucleic 
Acids Res 2009; 37: W202-W208, Web Server, DOl: https://doi.org/10.1093/na r/gkp335.

42. Jiang YZ, Duan YJ, Yin J, Ye SL, Zhu JR, Zhang FQ, Lu WX, Fan D, Luo KM. Genome-wide identification and characterization of the Populus WRKY transcription factor family and analysis of their expression in response to biotic and abiotic stresses. J Exp Bot. 2014;65(22):6629-44. https://doi.org/1 0.1093/jxb/eru381.

43. Machowski EE, Dawes S, Mizrahi V. TB tools to tell the tale-molecular genetic methods for mycobacterial research. Int J Biochem Cell Biol 2005; 37(1): 0-68, 54, DOI: https://doi.org/10.1016/j.biocel.2004.06.012.

44. Cao J, Huang JL, Yang YP, Hu XY. Analyses of the oligopeptide transporter gene family in poplar and grape. BMC Genomics. 2011;12(1):465. https://doi. org/10.1186/1471-2164-12-465.

45. Koressaar T, Lepamets M, Kaplinski L, Raime K, Andreson R, Remm M. Primer3_masker: integrating masking of template sequence with primer design software. Bioinformatics. 2018;34(11):1937-8. https://doi.org/10.1093/ bioinformatics/bty036.

46. Fan C, Ma J, Guo Q, Li X, Wang H, Lu M. Selection of reference genes for quantitative real-time PCR in bamboo (Phyllostachys edulis). PLoS One. 2013; 8(2):e56573. https://doi.org/10.1371/journal.pone.0056573.

\section{Publisher's Note}

Springer Nature remains neutral with regard to jurisdictional claims in published maps and institutional affiliations.

Ready to submit your research? Choose BMC and benefit from:

- fast, convenient online submission

- thorough peer review by experienced researchers in your field

- rapid publication on acceptance

- support for research data, including large and complex data types

- gold Open Access which fosters wider collaboration and increased citations

- maximum visibility for your research: over $100 \mathrm{M}$ website views per year

At BMC, research is always in progress.

Learn more biomedcentral.com/submissions 